\title{
VERIFICATION SURVEY OF FINAL GRIDS AT THE DAVID WITHERSPOON, INC. 1630 SITE KNOXVILLE, TENNESSEE
}

\author{
Prepared by \\ P. C. Weaver \\ Oak Ridge Institute for Science and Education \\ Oak Ridge, Tennessee 37831-0117 \\ Prepared for the \\ U.S. Department of Energy \\ DOE CONTRACT NO. DE-AC05-06OR23100
}

\section{LETTER REPORT}

FEBRUARY 2009

Approved for public release; further dissemination unlimited. 


\section{LETTER REPORT \\ VERIFICATION SURVEY OF FINAL GRIDS AT THE \\ DAVID WITHERSPOON, INC. 1630 SITE \\ KNOXVILLE, TENNESSEE}

\section{INTRODUCTION}

The David Witherspoon Incorporated 1630 Site (DWI 1630) received, processed, stored, and shipped scrap metal, including metal contaminated with radiological and chemical contaminants. Past site characterization activities identified numerous radiological and chemical contaminants, including uranium, thorium, heavy metals, polychlorinated biphenyls (PCBs), asbestos, and organic compounds. The contaminants have been noted in various media on the site, including debris, soil, sediment, groundwater, and surface water. There were no buildings located in the subject areas during site operations. The DOE's decommissioning contractor, Bechtel Jacobs Company, LLC. (BJC), has performed characterization surveys, subsequent remediation, and final status surveys and sampling within the subject area. The areas have not been backfilled pending results from the Independent Verification (IV) survey.

The U.S. Department of Energy (DOE) requested that the Oak Ridge Institute for Science and Education (ORISE) conduct verification surveys of grids at the DWI 1630 Site in Knoxville, Tennessee. During the period from October to December, 2008, an independent verification team (IVT) from ORISE, conducted verification activities in whole and partial grids, as completed by BJC. ORISE site activities included gamma surface scans and soil sampling within 33 grids; G11 through G14; H11 through H15; X14, X15, X19, and X21; J13 through J15 and J17 through J21; K7 through K9 and K13 through K15; L13 through L15; and M14 through M16 (Figure 1).

\section{PROCEDURES}

Site verification activities were performed in accordance with a Project-Specific Plan (PSP) submitted to and approved by DOE (ORISE 2007) and the ORISE Survey Procedures and the ORAU Quality Program Manuals (ORISE 2008a and ORAU 2007). The PSP was enhanced by implementing a random sample design process. Grid boundaries for the specified survey area were obtained from maps or coordinates provided by the contractor. This information aided in developing a map using ArcGIS software that was uploaded to a global positioning system (GPS). The GPS was used to collect measurement information during gamma radiation walkover surface scans so that gamma radiation count rates could be geo-referenced to a map position.

ORISE performed medium to high density gamma scans over approximately $75 \%$ or greater of accessible surfaces in the subject grids (Figure 2). Scans were performed using both 1.25 inch by 1.5 inch and 2 inch by 2 inch sodium iodide $(\mathrm{NaI})$ scintillation detectors coupled to ratemeters with audible output. The a priori scan MDC was approximately $115 \mathrm{pCi} / \mathrm{g}$ for the primary radionuclide of concern—uranium (U-238)—as estimated from Table 6.4 of NUREG-1507(NRC 1997).

All referenced map information was uploaded into VSP version 5 or higher software to develop a sampling design plan (PNNL 2007). The VSP software was used to determine the number of samples required that would provide an adequate statistical representation of the area based on the assumed mean concentration and variability of U-238, and to generate random soil sampling locations. At least one surface soil sample $(0$ to $0.15 \mathrm{~m})$ per grid was collected for radiological 
analyses. Each soil sample coordinate location was uploaded into a hand-held GPS unit. Additional random sampling locations were generated using VSP and used in place of original sample locations that were inaccessible.

Static gamma radiation measurements were recorded prior to the collection of a radiological soil sample. A follow-up gamma measurement was made after the sample had been collected to determine if the gamma radiation level increased with depth. An increase in radiation level required additional investigation and sampling.

ORISE also obtained soil samples in select grids to analyze for PCBs and the heavy metals lead (Pb) and cadmium $(\mathrm{Cd})$. Chemical samples were collected either when ORISE was notified by the contractor that the area contained residual chemical contamination or if historical information identified the area as having a potential for chemical contamination.

\section{SAMPLE ANALYSIS AND DATA INTERPRETATION}

The soil samples were returned to the ORISE laboratory in Oak Ridge, TN for analysis and interpretation. Samples were analyzed by gamma spectroscopy in accordance with the ORISE Laboratory Procedures Manual (ORISE 2008b). The soil sample spectra were reviewed for the photopeaks associated with U-238, Ra-226, and Th-232, as well as other gamma-emitting radionuclides. Alpha spectroscopy analysis was used to quantify individual uranium and thorium isotopes. All analytical results were quantified in units of picocuries per gram $(\mathrm{pCi} / \mathrm{g})$.

Soil samples collected for PCBs and metals $(\mathrm{Pb}$ and $\mathrm{Cd}$ ) were analyzed by Lionville Laboratory, Inc. (LVLI) in accordance with approved U.S. Environmental Protection Agency (EPA) procedures. The results were reported in units of micrograms per kilogram $(\mu \mathrm{g} / \mathrm{kg})$ and converted to units of milligrams per kilogram ( $\mathrm{mg} / \mathrm{kg})$.

\section{FINDINGS AND RESULTS}

The ambient background radiation levels were not subtracted from data reported. Gamma radiation count rates ranged from 6,000 to 25,000 cpm (Figures 2 to 7). The highest location identified was found outside of Grid L13 in Grid L12 during the final verification survey. It appeared to be a small localized spot of higher radioactivity (Figure 7). ORISE collected 37 random samples and one judgmental sample for radiological analyses and 13 samples for chemical analyses at the randomly generated radiological sample locations (Figure 8). The one judgmental sample was collected from the location of elevated direct gamma radiation identified during the surface scans.

Gamma spectroscopy results for samples S059 through S097 have been provided in Table 1. Sample number S065 was omitted during sampling due to the area being inaccessible and S097 was the only judgmental sample collected. The predominant radionuclide at the site is U-238 with concentrations determined by gamma spectroscopy ranging from 0.91 to $470 \mathrm{pCi} / \mathrm{g}$. The highest U-238 concentration identified was located in Grid L12 soil sample S097. The next highest concentration was found at sample location S091 (Grid M15) with a concentration of $50.3 \mathrm{pCi} / \mathrm{g}$. The Ra-226 concentrations ranged from 0.37 to $46.5 \mathrm{pCi} / \mathrm{g}$ and the Th- 232 concentrations ranged from 0.32 to $1.68 \mathrm{pCi} / \mathrm{g}$. The spectra were also reviewed for other identifiable radionuclide photopeaks, which have been included in Table 1. Total $U$ and total Th ranged from 1.93 to $960 \mathrm{pCi} / \mathrm{g}$ and -1.70 to $3.14 \mathrm{pCi} / \mathrm{g}$, respectively. Alpha spectroscopy was conducted on a sample batch that included soil 
samples S066 through S083 (Figure 8). The total thorium and total uranium concentrations ranged from 2.35 to $3.17 \mathrm{pCi} / \mathrm{g}$ and 2.22 to $11.22 \mathrm{pCi} / \mathrm{g}$, respectively (Table 2). All sample data were compared to the $500 \mathrm{pCi} / \mathrm{g}$ "hot-spot" criterion for U-238, as specified in the PSP (ORISE 2007a).

PCB sample results have been provided in Table 3. Data were reviewed for the primary PCB isomer contaminant, Aroclor 1254. Additionally, measurable concentrations of the PCB isomers Aroclor 1248 and Aroclor 1260 were also reported. Total PCBs, which usually includes the isomers Aroclor 1254 and 1260; have been reported in Table 3. However, Aroclor 1248 was measured in concentrations greater than the minimum detectable level and was therefore, included in the total PCB concentrations reported. Aroclor 1254 concentrations ranged from 0.01 to $0.72 \mathrm{mg} / \mathrm{kg}$. Two samples exceeded the $0.87 \mathrm{mg} / \mathrm{kg}$ remediation levels (RLs) for total PCBs at 2.3 and $1.7 \mathrm{mg} / \mathrm{kg}$ in S068 (Grid H14) and S069 (Grid X15), respectively.

$\mathrm{Pb}$ and $\mathrm{Cd}$ results are reported in Table $4 \mathrm{~Pb}$ results were compared to the applicable site $\mathrm{RL}$ of $800 \mathrm{mg} / \mathrm{kg}$. Pb concentrations ranged form 19.2 to $108 \mathrm{mg} / \mathrm{kg}$, which are well below the RL. The highest concentration of $\mathrm{Pb}$ was sampled at location $\mathrm{S} 068$. In most samples, Cd results were below the minimum detection limit for the sample. Measurable Cd ranged from 0.13 to $0.83 \mathrm{mg} / \mathrm{kg}$. An $\mathrm{RL}$ for Cd has not been established for the site.

\section{CONCLUSIONS}

It is ORISE's position that the concentration of the radionuclides of concern for the subject grids, meet the release limits established for the DWI 1630 site. However, the highest concentration of U-238 based upon results from Gamma spectroscopy, was identified in the ORISE radiological sample result for sample $\mathrm{S} 097$ at $470 \mathrm{pCi} / \mathrm{g}$. This is within the hot spot criteria for the U-238, "hot spot" limit of $500 \mathrm{pCi} / \mathrm{g}$ and the total U concentration at $\mathrm{S} 097$ was $960 \mathrm{pCi} / \mathrm{g}$.

PCB concentrations at locations S068 and S069 were greater than the $0.87 \mathrm{mg} / \mathrm{kg} \mathrm{RL}$ at 2.3 and $1.7 \mathrm{mg} / \mathrm{kg}$, respectively. On average; however, PCB concentrations were below the RL. Pb concentrations were below the RL and Cd has no RL for the site; however, concentrations were low. 


\section{FIGURES}




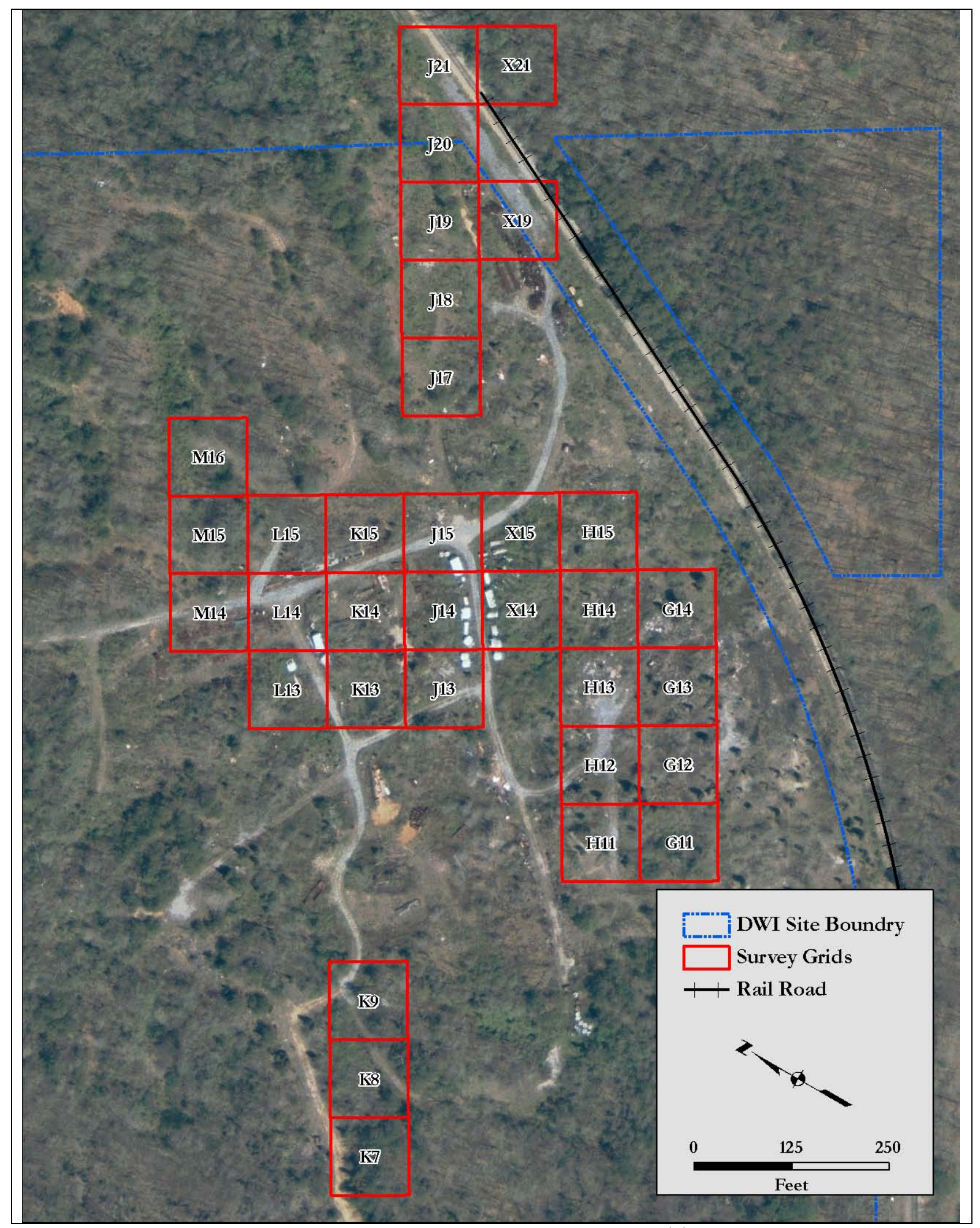

FIGURE 1: SURVEYED GRID UNITS 


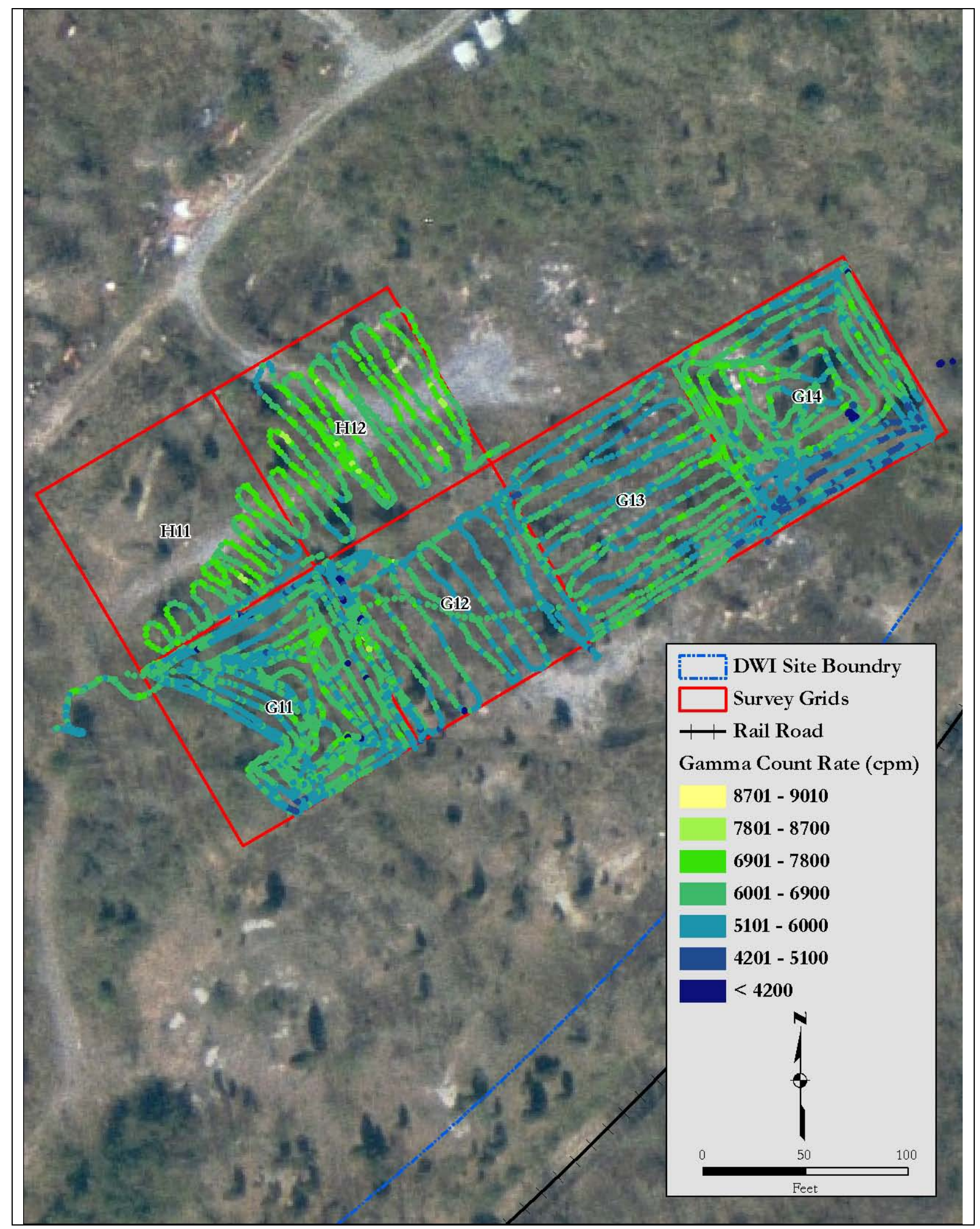

FIGURE 2: SODIUM IODIDE GAMMA WALKOVER SCANS GRIDS H11, H12 AND G11 THROUGH G13 


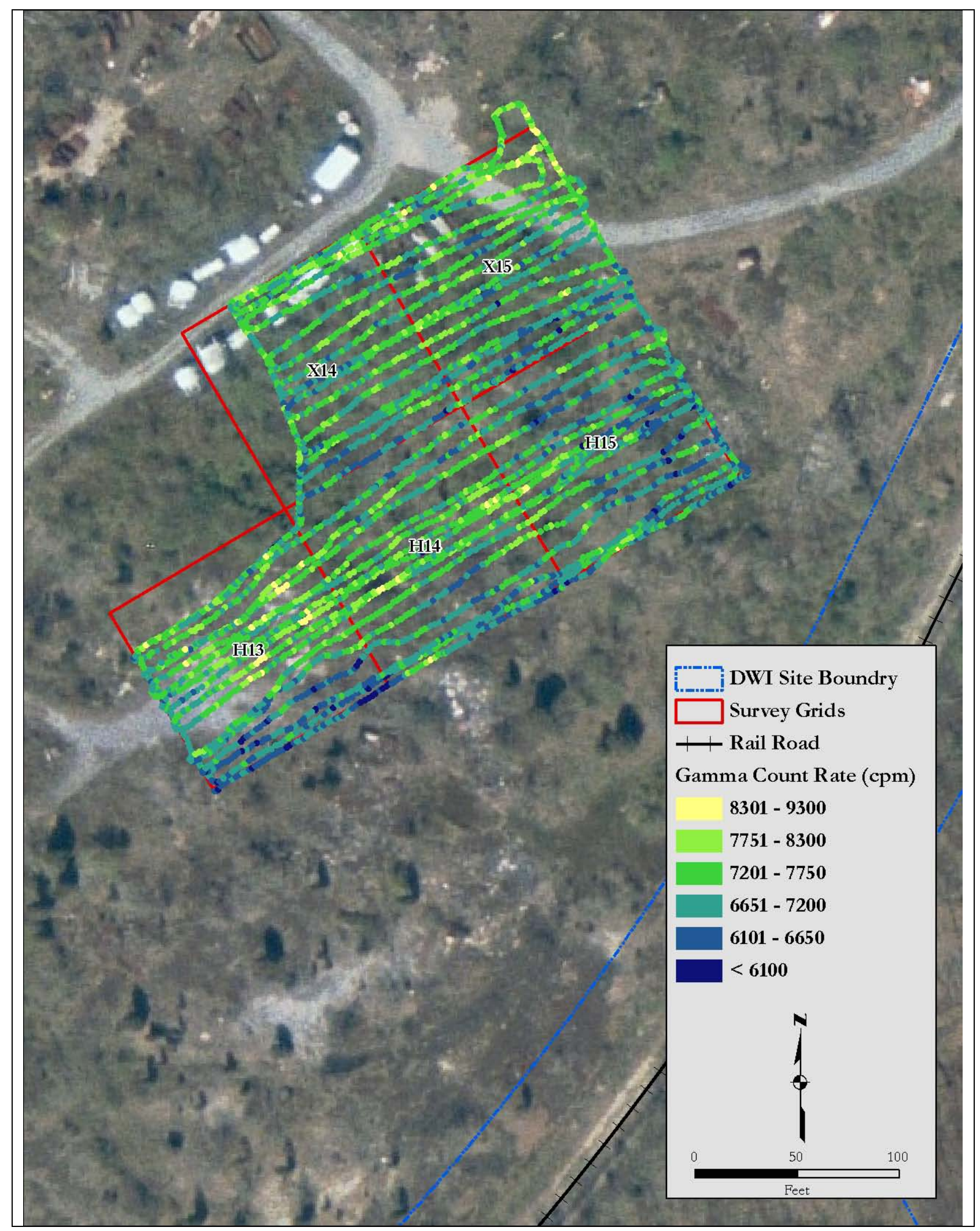

FIGURE 3: SODIUM IODIDE GAMMA WALKOVER SCANS

H13 THROUGH H15, X14 AND X15 


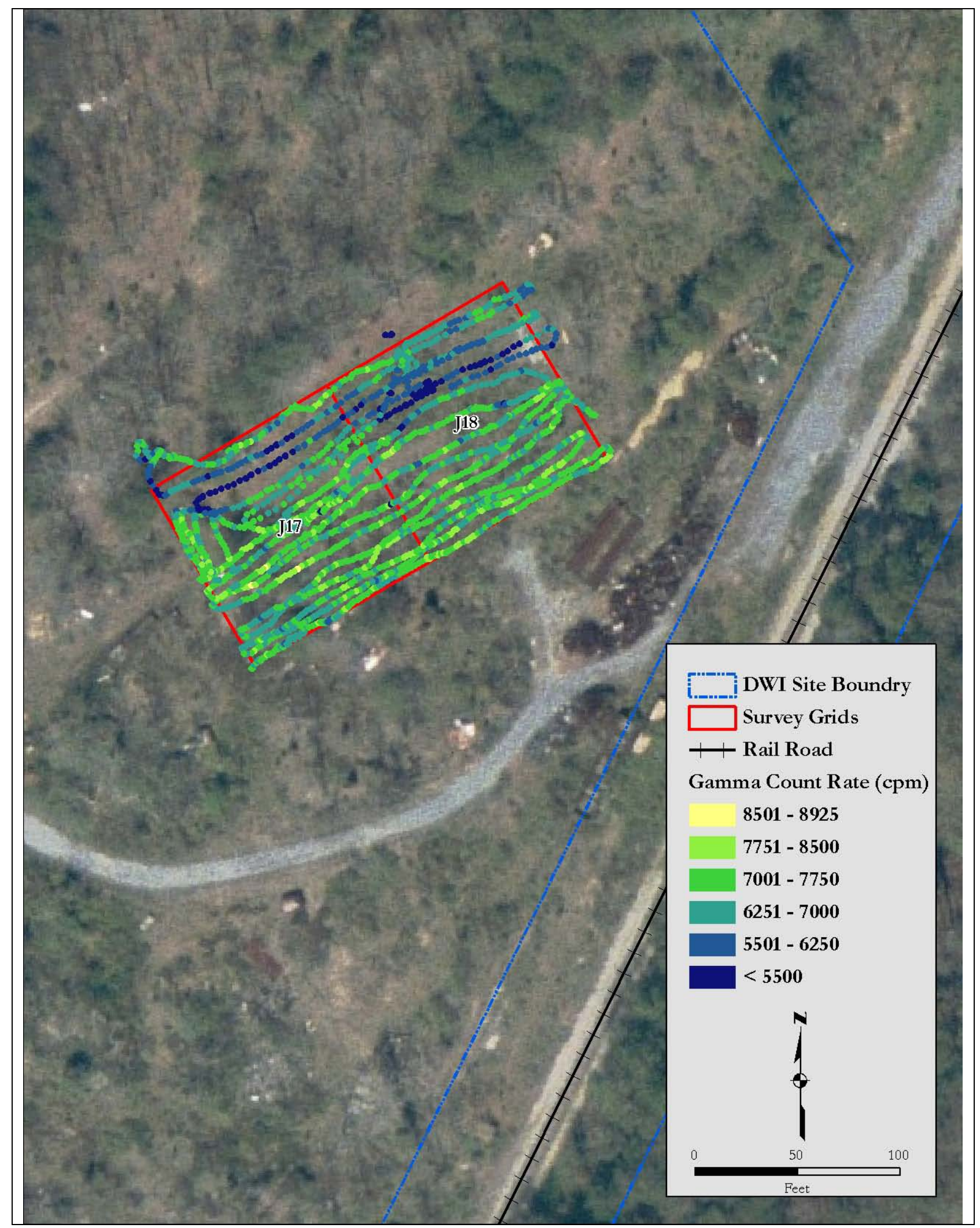

FIGURE 4: SODIUM IODIDE GAMMA WALKOVER SCANS GRIDS J17 AND J18 


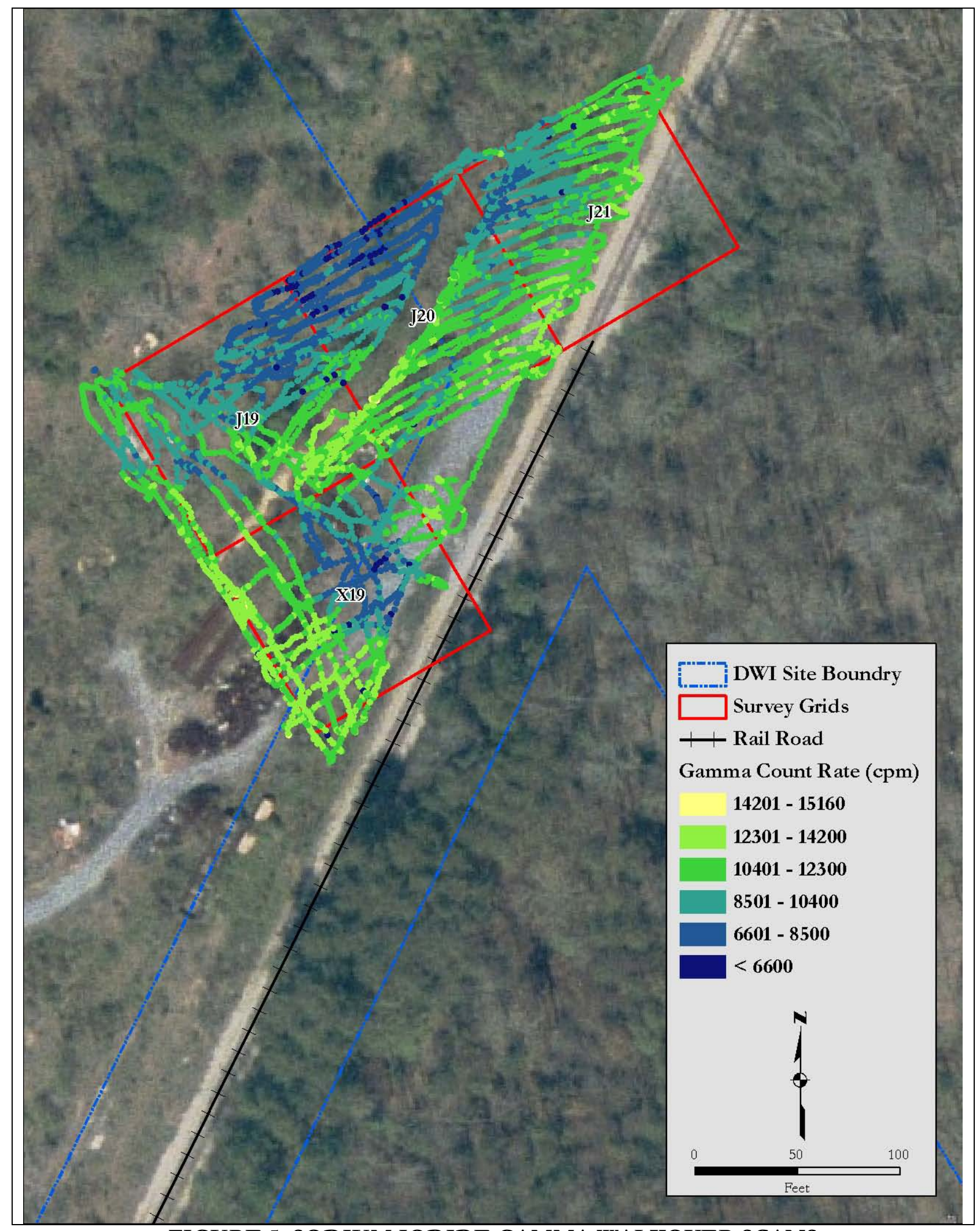

FIGURE 5: SODIUM IODIDE GAMMA WALKOVER SCANS GRIDS J19 THROUGH J21 AND GRID X19 


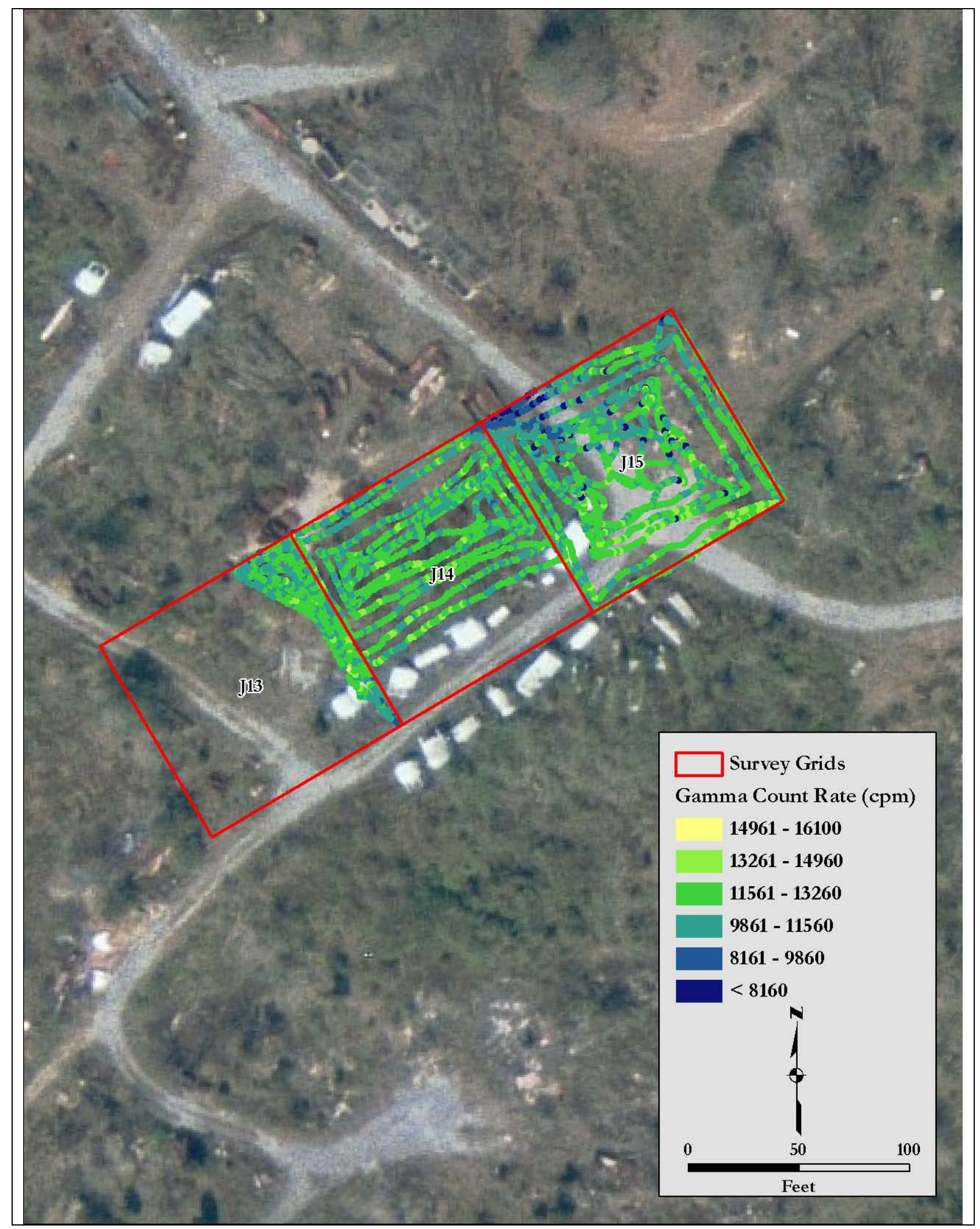

FIGURE 6: SODIUM IODIDE GAMMA WALKOVER SCANS GRIDS J13 THROUGH J15 


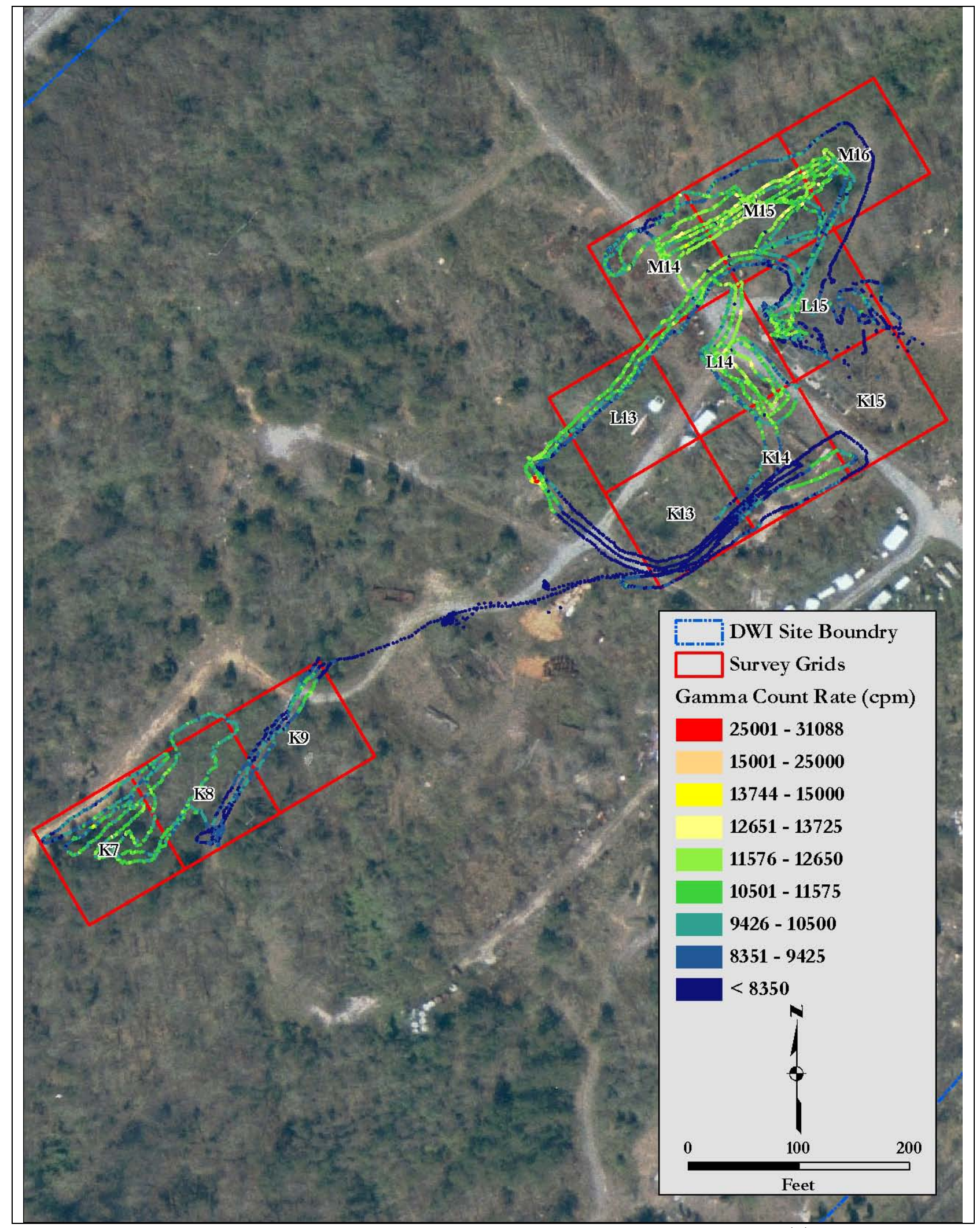

FIGURE 7: SODIUM IODIDE GAMMA WALKOVER SCANS GRIDS K7THROUGH K9, K13 THROUGH K15, L13 THROUGH L15, AND M14 THROUGH M16 


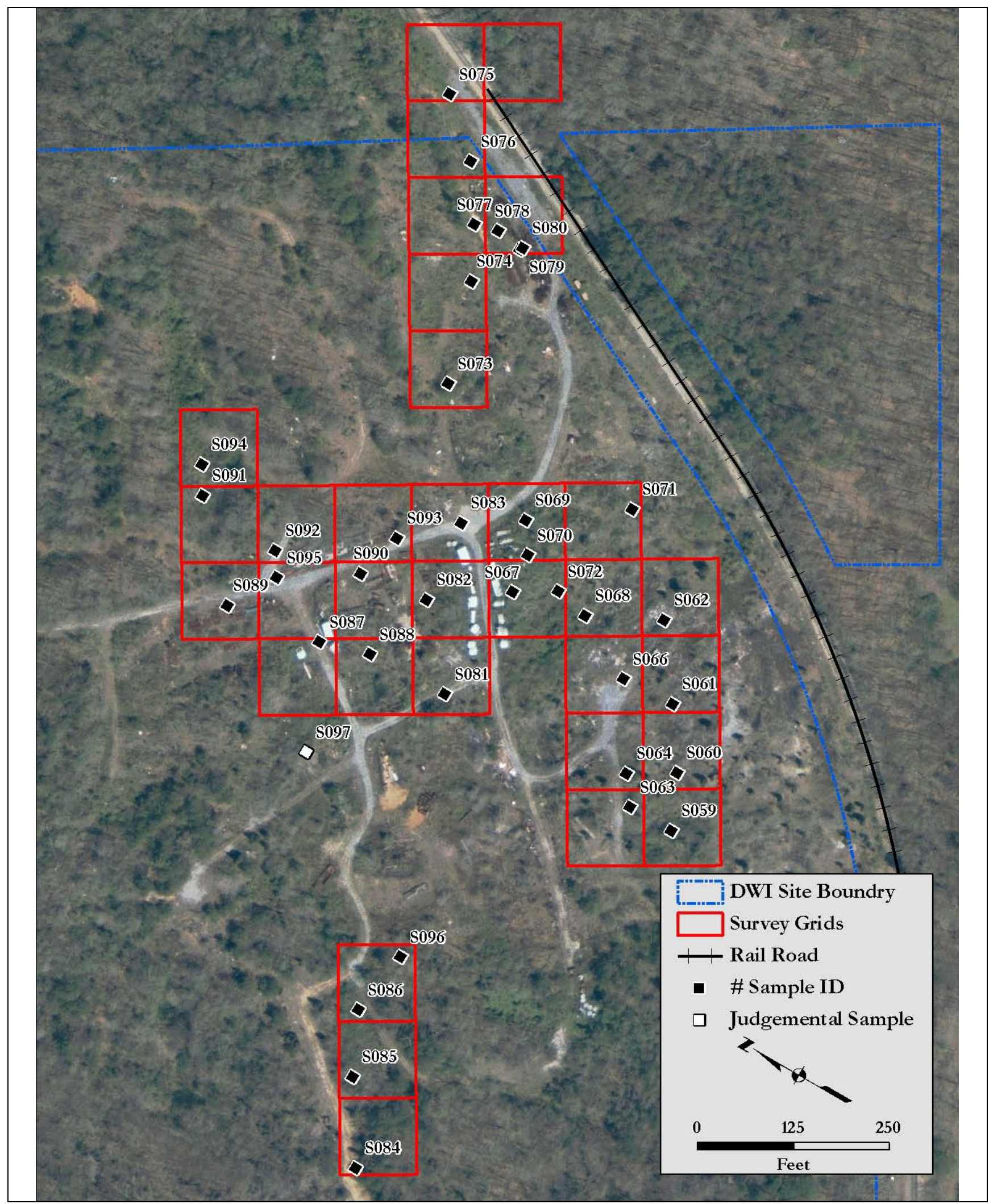

FIGURE 8: RANDOM AND JUDGMENTAL SAMPLE LOCATIONS 
TABLES 


\section{TABLE 1}

\section{RADIONUCLIDE CONCENTRATIONS IN SOIL BY GAMMA SPECTROSCOPY}

\section{DAVID WITHERSPOON, INC. 1630 SITE}

KNOXVILLE, TENNESSEE

\begin{tabular}{|c|c|c|c|c|c|c|c|c|}
\hline \multirow{2}{*}{$\begin{array}{c}\text { Sample } \\
\text { ID/Location }\end{array}$} & \multicolumn{8}{|c|}{ Radionuclide Concentration in Soil Samples (pCi/g) } \\
\hline & Cs-137 & Ra-226 & Th-228 & Th-232 & Total $\mathbf{T h}^{\mathrm{b}}$ & $\mathrm{U}-238$ & U-235 & Total Uc \\
\hline S059 & $0.00 \pm 0.04 \mathrm{~d}$ & $0.55 \pm 0.06$ & $1.08 \pm 0.09$ & $1.18 \pm 0.17$ & $2.26 \pm 0.19$ & $1.02 \pm 0.31$ & $0.10 \pm 0.14$ & $2.14 \pm 0.64$ \\
\hline S060 & $0.02 \pm 0.01$ & $0.64 \pm 0.07$ & $1.16 \pm 0.10$ & $1.57 \pm 0.23$ & $2.73 \pm 0.25$ & $1.39 \pm 0.40$ & $0.08 \pm 0.07$ & $2.86 \pm 0.80$ \\
\hline S061 & $0.02 \pm 0.02$ & $0.64 \pm 0.06$ & $1.27 \pm 0.10$ & $1.30 \pm 0.17$ & $2.57 \pm 0.20$ & $1.53 \pm 0.29$ & $0.22 \pm 0.16$ & $3.28 \pm 0.60$ \\
\hline S062 & $0.02 \pm 0.01$ & $0.64 \pm 0.05$ & $1.14 \pm 0.09$ & $1.30 \pm 0.16$ & $2.44 \pm 0.18$ & $1.45 \pm 0.25$ & $0.04 \pm 0.07$ & $2.94 \pm 0.50$ \\
\hline S063 & $0.17 \pm 0.03$ & $0.72 \pm 0.07$ & $1.34 \pm 0.11$ & $1.43 \pm 0.20$ & $2.77 \pm 0.23$ & $1.46 \pm 0.34$ & $0.08 \pm 0.06$ & $3.00 \pm 0.68$ \\
\hline S064 & $0.04 \pm 0.04$ & $0.51 \pm 0.06$ & $1.02 \pm 0.09$ & $1.27 \pm 0.18$ & $2.29 \pm 0.20$ & $1.05 \pm 0.33$ & $0.13 \pm 0.21$ & $2.23 \pm 0.69$ \\
\hline S066 & $0.04 \pm 0.02$ & $0.64 \pm 0.07$ & $1.31 \pm 0.11$ & $1.50 \pm 0.22$ & $2.81 \pm 0.25$ & $7.88 \pm 0.79$ & $0.33 \pm 0.09$ & $16.1 \pm 1.6$ \\
\hline S067 & $0.04 \pm 0.05$ & $0.92 \pm 0.09$ & $1.36 \pm 0.12$ & $1.50 \pm 0.22$ & $2.86 \pm 0.25$ & $1.72 \pm 0.45$ & $0.05 \pm 0.27$ & $3.49 \pm 0.94$ \\
\hline S068 & $0.07 \pm 0.02$ & $0.84 \pm 0.08$ & $1.53 \pm 0.12$ & $1.50 \pm 0.21$ & $3.03 \pm 0.24$ & $1.52 \pm 0.35$ & $-0.02 \pm 0.22$ & $3.02 \pm 0.73$ \\
\hline S069 & $0.02 \pm 0.02$ & $0.59 \pm 0.07$ & $1.11 \pm 0.10$ & $1.29 \pm 0.20$ & $2.40 \pm 0.22$ & $4.91 \pm 0.60$ & $0.16 \pm 0.07$ & $10.0 \pm 1.2$ \\
\hline S070 & $0.04 \pm 0.02$ & $0.70 \pm 0.06$ & $1.40 \pm 0.11$ & $1.44 \pm 0.20$ & $2.84 \pm 0.23$ & $1.74 \pm 0.34$ & $0.10 \pm 0.06$ & $3.58 \pm 0.68$ \\
\hline S071 & $0.02 \pm 0.01$ & $0.68 \pm 0.07$ & $1.13 \pm 0.10$ & $1.18 \pm 0.17$ & $2.31 \pm 0.20$ & $1.31 \pm 0.35$ & $0.14 \pm 0.08$ & $2.76 \pm 0.70$ \\
\hline S072 & $0.08 \pm 0.02$ & $0.79 \pm 0.08$ & $1.48 \pm 0.13$ & $1.42 \pm 0.21$ & $2.90 \pm 0.25$ & $1.93 \pm 0.43$ & $0.09 \pm 0.08$ & $3.95 \pm 0.86$ \\
\hline S073 & $-0.03 \pm 0.07$ & $0.72 \pm 0.09$ & $1.36 \pm 0.12$ & $1.58 \pm 0.24$ & $2.94 \pm 0.27$ & $1.95 \pm 0.49$ & $0.09 \pm 0.31$ & $4.0 \pm 1.0$ \\
\hline S074 & $-0.01 \pm 0.04$ & $0.57 \pm 0.06$ & $1.17 \pm 0.09$ & $1.23 \pm 0.17$ & $2.40 \pm 0.19$ & $1.55 \pm 0.32$ & $0.19 \pm 0.18$ & $3.29 \pm 0.66$ \\
\hline S075 & $-0.03 \pm 0.05$ & $0.65 \pm 0.07$ & $1.39 \pm 0.12$ & $1.54 \pm 0.22$ & $2.93 \pm 0.25$ & $2.31 \pm 0.46$ & $0.15 \pm 0.11$ & $4.77 \pm 0.93$ \\
\hline S076 & $-0.02 \pm 0.07$ & $0.61 \pm 0.08$ & $1.29 \pm 0.12$ & $1.56 \pm 0.24$ & $2.85 \pm 0.27$ & $2.92 \pm 0.55$ & $0.11 \pm 0.08$ & $6.0 \pm 1.1$ \\
\hline S077 & $-0.03 \pm 0.05$ & $0.64 \pm 0.07$ & $1.41 \pm 0.12$ & $1.51 \pm 0.21$ & $2.92 \pm 0.24$ & $1.49 \pm 0.39$ & $0.14 \pm 0.09$ & $3.12 \pm 0.79$ \\
\hline S078 & $0.02 \pm 0.05$ & $0.65 \pm 0.07$ & $1.46 \pm 0.13$ & $1.68 \pm 0.24$ & $3.14 \pm 0.27$ & $1.23 \pm 0.35$ & $0.02 \pm 0.17$ & $2.48 \pm 0.72$ \\
\hline S079 & $0.02 \pm 0.06$ & $0.63 \pm 0.07$ & $1.35 \pm 0.12$ & $1.44 \pm 0.21$ & $2.79 \pm 0.24$ & $1.48 \pm 0.44$ & $0.08 \pm 0.12$ & $3.04 \pm 0.89$ \\
\hline S080 & $0.00 \pm 0.04$ & $0.68 \pm 0.07$ & $1.49 \pm 0.12$ & $1.65 \pm 0.21$ & $3.14 \pm 0.24$ & $1.38 \pm 0.31$ & $0.10 \pm 0.07$ & $2.86 \pm 0.62$ \\
\hline
\end{tabular}




\section{TABLE 1 (Continued)}

\section{RADIONUCLIDE CONCENTRATIONS IN SOIL}

\section{BY GAMMA SPECTROSCOPY}

DAVID WITHERSPOON, INC. 1630 SITE KNOXVILLE, TENNESSEE

\begin{tabular}{|c|c|c|c|c|c|c|c|c|}
\hline \multirow{2}{*}{$\begin{array}{l}\text { Sample } \\
\text { ID/Location }\end{array}$} & \multicolumn{8}{|c|}{ Radionuclide Concentration in Soil Samples (pCi/g) } \\
\hline & Cs-137 & Ra-226 & Th-228 & Th-232 & Total Th${ }^{b}$ & U-238 & U-235 & Total Uc \\
\hline S081 & $-0.03 \pm 0.05$ & $0.66 \pm 0.07$ & $1.34 \pm 0.12$ & $1.39 \pm 0.21$ & $2.73 \pm 0.24$ & $2.06 \pm 0.45$ & $0.15 \pm 0.09$ & $4.27 \pm 0.90$ \\
\hline S082 & $0.05 \pm 0.06$ & $0.66 \pm 0.08$ & $1.32 \pm 0.12$ & $1.43 \pm 0.22$ & $2.75 \pm 0.25$ & $2.92 \pm 0.55$ & $0.21 \pm 0.10$ & $6.0 \pm 1.1$ \\
\hline S083 & $0.02 \pm 0.01$ & $0.60 \pm 0.07$ & $1.30 \pm 0.11$ & $1.39 \pm 0.20$ & $2.69 \pm 0.23$ & $1.70 \pm 0.43$ & $0.14 \pm 0.09$ & $3.54 \pm 0.86$ \\
\hline S084 & $0.06 \pm 0.03$ & $0.66 \pm 0.08$ & $1.35 \pm 0.12$ & $1.36 \pm 0.22$ & $2.71 \pm 0.25$ & $1.53 \pm 0.45$ & $0.08 \pm 0.12$ & $3.14 \pm 0.91$ \\
\hline S085 & $0.02 \pm 0.01$ & $0.57 \pm 0.05$ & $1.21 \pm 0.10$ & $1.34 \pm 0.17$ & $2.55 \pm 0.20$ & $1.23 \pm 0.25$ & $0.13 \pm 0.16$ & $2.59 \pm 0.52$ \\
\hline S086 & $0.11 \pm 0.02$ & $0.69 \pm 0.07$ & $1.16 \pm 0.10$ & $1.25 \pm 0.18$ & $2.41 \pm 0.21$ & $0.91 \pm 0.28$ & $0.13 \pm 0.07$ & $1.95 \pm 0.56$ \\
\hline S087 & $0.08 \pm 0.05$ & $0.74 \pm 0.08$ & $1.37 \pm 0.12$ & $1.52 \pm 0.23$ & $2.89 \pm 0.26$ & $1.01 \pm 0.42$ & $-0.09 \pm 0.29$ & $1.93 \pm 0.89$ \\
\hline S088 & $-0.08 \pm 0.02$ & $0.52 \pm 0.06$ & $1.25 \pm 0.11$ & $1.50 \pm 0.23$ & $2.75 \pm 0.25$ & $8.06 \pm 0.73$ & $0.37 \pm 0.09$ & $16.5 \pm 1.5$ \\
\hline S089 & $0.04 \pm 0.02$ & $0.81 \pm 0.08$ & $1.41 \pm 0.12$ & $1.67 \pm 0.22$ & $3.08 \pm 0.25$ & $1.37 \pm 0.39$ & $0.16 \pm 0.08$ & $2.90 \pm 0.78$ \\
\hline S090 & $0.04 \pm 0.02$ & $0.60 \pm 0.07$ & $0.87 \pm 0.09$ & $0.99 \pm 0.18$ & $1.86 \pm 0.20$ & $6.05 \pm 0.65$ & $0.27 \pm 0.09$ & $12.4 \pm 1.3$ \\
\hline S091 & $-0.28 \pm 0.13$ & $46.5 \pm 2.7$ & $-2.02 \pm 0.22$ & $0.32 \pm 0.66$ & $-1.70 \pm 0.70$ & $50.3 \pm 3.8$ & $3.16 \pm 0.46$ & $103.8 \pm 7.6$ \\
\hline S092 & $0.02 \pm 0.01$ & $0.65 \pm 0.06$ & $0.81 \pm 0.07$ & $0.88 \pm 0.13$ & $1.69 \pm 0.15$ & $1.29 \pm 0.25$ & $0.09 \pm 0.01$ & $2.67 \pm 0.50$ \\
\hline S093 & $0.02 \pm 0.01$ & $0.37 \pm 0.04$ & $0.41 \pm 0.04$ & $0.40 \pm 0.07$ & $0.81 \pm 0.08$ & $2.39 \pm 0.27$ & $0.15 \pm 0.04$ & $4.93 \pm 0.54$ \\
\hline S094 & $0.13 \pm 0.02$ & $0.68 \pm 0.07$ & $1.06 \pm 0.09$ & $1.19 \pm 0.17$ & $2.25 \pm 0.19$ & $1.10 \pm 0.34$ & $0.08 \pm 0.07$ & $2.28 \pm 0.68$ \\
\hline S095 & $0.03 \pm 0.02$ & $0.83 \pm 0.08$ & $1.36 \pm 0.12$ & $1.33 \pm 0.20$ & $2.69 \pm 0.23$ & $1.55 \pm 0.43$ & $-0.06 \pm 0.13$ & $3.04 \pm 0.87$ \\
\hline S096 & $0.01 \pm 0.02$ & $0.54 \pm 0.05$ & $0.96 \pm 0.08$ & $0.97 \pm 0.13$ & $1.93 \pm 0.15$ & $1.10 \pm 0.25$ & $0.05 \pm 0.07$ & $2.25 \pm 0.50$ \\
\hline S097e & $0.32 \pm 0.05$ & $0.73 \pm 0.08$ & $1.11 \pm 0.10$ & $1.17 \pm 0.18$ & $2.28 \pm 0.21$ & $470 \pm 30$ & $22.7 \pm 1.3$ & $960 \pm 60$ \\
\hline
\end{tabular}

${ }^{a}$ Refer to Figure 8

'Total thorium concentrations determined as follows: (Th-228 + Th-232).

cTotal uranium concentrations determined as follows: (U-238 x 2) + U-235.

dUncertainties represent the $95 \%$ confidence level based on total propagated uncertainties.

eJudgmental sample 
TABLE 2

RADIONUCLIDE CONCENTRATIONS IN SOIL BY ALPHA SPECTROSCOPY

\section{DAVID WITHERSPOON, INC. 1630 SITE} KNOXVILLE, TENNESSEE

\begin{tabular}{|c|c|c|c|c|c|c|c|}
\hline $\begin{array}{c}\text { Sample } \\
\text { ID/Location }\end{array}$ & \multicolumn{7}{|c|}{ Radionuclide Concentration in Soil Samples (pCi/g) } \\
\hline S066 & $1.27 \pm 0.19^{\mathrm{d}}$ & $1.27 \pm 0.19$ & $2.54 \pm 0.26$ & $5.43 \pm 0.44$ & $0.27 \pm 0.05$ & $5.36 \pm 0.43$ & $11.05 \pm 0.61$ \\
\hline S067 & $1.48 \pm 0.23$ & $1.34 \pm 0.21$ & $2.82 \pm 0.32$ & $1.57 \pm 0.16$ & $0.11 \pm 0.03$ & $1.60 \pm 0.16$ & $3.28 \pm 0.23$ \\
\hline S068 & $1.47 \pm 0.22$ & $1.40 \pm 0.21$ & $2.87 \pm 0.30$ & $1.18 \pm 0.13$ & $0.06 \pm 0.02$ & $1.38 \pm 0.14$ & $2.62 \pm 0.19$ \\
\hline S069 & $1.32 \pm 0.19$ & $1.20 \pm 0.18$ & $2.52 \pm 0.26$ & $5.30 \pm 0.43$ & $0.31 \pm 0.06$ & $5.60 \pm 0.45$ & $11.22 \pm 0.62$ \\
\hline S071 & $1.23 \pm 0.21$ & $1.11 \pm 0.19$ & $2.35 \pm 0.28$ & $1.28 \pm 0.14$ & $0.07 \pm 0.03$ & $1.31 \pm 0.15$ & $2.65 \pm 0.21$ \\
\hline S072 & $1.43 \pm 0.22$ & $1.25 \pm 0.19$ & $2.68 \pm 0.29$ & $1.38 \pm 0.14$ & $0.08 \pm 0.03$ & $1.46 \pm 0.15$ & $2.92 \pm 0.21$ \\
\hline S073 & $1.30 \pm 0.19$ & $1.31 \pm 0.19$ & $2.61 \pm 0.27$ & $2.16 \pm 0.20$ & $0.13 \pm 0.04$ & $2.00 \pm 0.19$ & $4.29 \pm 0.28$ \\
\hline S074 & $1.19 \pm 0.19$ & $1.22 \pm 0.18$ & $2.41 \pm 0.26$ & $1.27 \pm 0.13$ & $0.08 \pm 0.03$ & $1.39 \pm 0.14$ & $2.74 \pm 0.19$ \\
\hline S078 & $1.55 \pm 0.21$ & $1.53 \pm 0.21$ & $3.07 \pm 0.30$ & $1.09 \pm 0.12$ & $0.06 \pm 0.02$ & $1.20 \pm 0.13$ & $2.34 \pm 0.17$ \\
\hline S079 & $1.55 \pm 0.22$ & $1.39 \pm 0.20$ & $2.94 \pm 0.29$ & $1.43 \pm 0.15$ & $0.05 \pm 0.02$ & $1.68 \pm 0.16$ & $3.16 \pm 0.22$ \\
\hline S080 & $1.55 \pm 0.22$ & $1.63 \pm 0.23$ & $3.17 \pm 0.32$ & $1.19 \pm 0.13$ & $0.09 \pm 0.03$ & $1.30 \pm 0.13$ & $2.58 \pm 0.19$ \\
\hline S081 & $1.40 \pm 0.20$ & $1.25 \pm 0.18$ & $2.65 \pm 0.28$ & $1.08 \pm 0.09$ & $0.07 \pm 0.39$ & $1.54 \pm 0.08$ & $2.68 \pm 0.17$ \\
\hline S082 & $1.25 \pm 0.18$ & $1.43 \pm 0.21$ & $2.68 \pm 0.28$ & $1.60 \pm 0.08$ & $0.07 \pm 0.38$ & $2.21 \pm 0.07$ & $3.88 \pm 0.20$ \\
\hline S083 & $1.54 \pm 0.22$ & $1.61 \pm 0.23$ & $3.15 \pm 0.32$ & $1.06 \pm 0.10$ & $0.06 \pm 0.03$ & $1.10 \pm 0.10$ & $2.22 \pm 0.14$ \\
\hline
\end{tabular}

${ }^{a}$ Refer to Figure 8.

bTotal thorium concentrations determined as follows: (Th-228 + Th-232).

cTotal uranium concentrations determined as follows: (U-234 + U-235 + U238).

dUncertainties represent the $95 \%$ confidence level based on total propagated uncertainties. 


\begin{tabular}{|c|c|c|c|c|}
\hline & & ABLE 3 & & \\
\hline & PCB CON & JTRATIONS & SOIL & \\
\hline & DAVID WIT & SPOON, INC & 630 SITE & \\
\hline $\begin{array}{c}\text { Sample ID/ } \\
\text { Location }^{\mathrm{a}}\end{array}$ & $\begin{array}{l}\text { Aroclor } 1254 \\
(\mathrm{mg} / \mathrm{kg})\end{array}$ & $\begin{array}{c}\text { Aroclor } 1260 \\
(\mathrm{mg} / \mathrm{kg})\end{array}$ & $\begin{array}{c}\text { Aroclor } 1248 \\
(\mathrm{mg} / \mathrm{kg})\end{array}$ & $\begin{array}{c}\text { Total } \\
(\mathrm{mg} / \mathrm{kg})\end{array}$ \\
\hline 059 & $0.015 \mathrm{U}^{\mathrm{b}}$ & $0.015 \mathrm{U}$ & $0.015 \mathrm{U}$ & $0.045 \mathrm{U}$ \\
\hline 060 & $0.015 \mathrm{U}$ & $0.015 \mathrm{U}$ & $0.015 \mathrm{U}$ & $0.046 \mathrm{U}$ \\
\hline 061 & 0.027 & $0.011 \mathrm{~J}^{\mathrm{c}}$ & $0.015 \mathrm{U}$ & $0.037 \mathrm{~J}$ \\
\hline 062 & 0.051 & $0.013 \mathrm{~J}$ & 0.055 & 0.120 \\
\hline 063 & 0.051 & 0.017 & 0.077 & 0.140 \\
\hline 064 & 0.030 & 0.033 & 0.059 & 0.120 \\
\hline 066 & 0.240 & 0.170 & 0.230 & 0.810 \\
\hline 067 & 0.190 & 0.088 & 0.330 & 0.610 \\
\hline 068 & 0.620 & 0.420 & 1.300 & 2.300 \\
\hline 069 & 0.720 & 0.230 & 0.750 & 1.700 \\
\hline 071 & 0.130 & 0.075 & 0.220 & 0.430 \\
\hline 073 & 0.033 & 0.160 & $0.009 \mathrm{~J}$ & 0.200 \\
\hline 074 & 0.081 & 0.099 & $0.015 \mathrm{U}$ & 0.200 \\
\hline
\end{tabular}

${ }^{a}$ Refer to Figure 8.

bPresent below detection limit. Refer to Appendix A for additional detail

'Indicates that the isomer was analyzed but not detected. Refer to Appendix A for additional detail 


\section{TABLE 4}

LEAD AND CADMIUM CONCENTRATIONS IN SOIL

DAVID WITHERSPOON, INC. 1630 SITE KNOXVILLE, TENNESSEE

\begin{tabular}{|c|c|c|}
\hline $\begin{array}{c}\text { Sample ID/ } \\
\text { Location }^{\text {a }}\end{array}$ & $\begin{array}{c}\text { Lead } \\
\text { (mg/ } \mathbf{k g})\end{array}$ & $\begin{array}{c}\text { Cadmium } \\
\text { (mg/ kg) }\end{array}$ \\
\hline 059 & 22.8 & 0.15 \\
\hline 060 & 25.6 & 0.16 \\
\hline 061 & 22.7 & 0.15 \\
\hline 062 & 40.9 & 0.46 \\
\hline 063 & 35.3 & 0.70 \\
\hline 065 & 19.2 & 0.21 \\
\hline 066 & 57.2 & 0.43 \\
\hline 067 & 25.7 & 0.13 \\
\hline 068 & 108 & 0.65 \\
\hline 069 & 33.1 & 0.83 \\
\hline 071 & 28.6 & 0.17 \\
\hline 073 & 33.0 & 0.13 \\
\hline 074 & 75.8 & 0.62 \\
\hline
\end{tabular}

${ }^{a}$ Refer to Figure 8. 


\section{REFERENCES}

Bechtel Jacobs Company LLC. (BJC). Sampling and Analysis Plan for Final Verification of the David Witherspoon, Inc. 1630 Site. Oak Ridge, Tennessee; December 2006.

Bechtel Jacobs Company LLC. Independent Verification Survey at the David Witherspoon Inc. 1630 Site. Oak Ridge, Tennessee; September 8, 2008.

Bechtel Jacobs Company LLC. Independent Verification Survey at the David Witherspoon Inc. 1630 Site. Oak Ridge, Tennessee; October, 2008.

Bechtel Jacobs Company LLC. Independent Verification Survey at the David Witherspoon Inc. 1630 Site. Oak Ridge, Tennessee; October, 2008.

Bechtel Jacobs Company LLC. Independent Verification Survey at the David Witherspoon Inc. 1630 Site. Oak Ridge, Tennessee; 2008.

Bechtel Jacobs Company LLC. Independent Verification Survey at the David Witherspoon Inc. 1630 Site. Oak Ridge, Tennessee; September 8, 2008.

Oak Ridge Associated Universities (ORAU). Quality Program Manual for the Independent Environmental Assessment and Verification Program. Oak Ridge, Tennessee; November 1, 2007.

Oak Ridge Institute for Science and Education (ORISE). Final Project-Specific Plan for Conducting In-Process Reviews and Independent Verification Surveys of Remedial Action Activities at the David Witherspoon, Inc. 1630 Site, Knoxville, Tennessee. Oak Ridge, Tennessee; May 17, 2007.

Oak Ridge Institute for Science and Education. Survey Procedures Manual for the Independent Environmental Assessment and Verification Program. Oak Ridge, Tennessee; May 1, 2008a.

Oak Ridge Institute for Science and Education. Laboratory Procedures Manual for the Independent Environmental Assessment and Verification Program. Oak Ridge, Tennessee; December 5, 2008b.

Pacific Northwest National Laboratory (PNNL). Visual Sample Plan Version 5.0 User's Guide. Richland, Washington, September 2007.

U.S. Department of Energy (DOE). Environment, Safety and Health Bulletin: A Guide to Good Practices for the Control and Release of Property. DOE/EH-0697. Washington, DC. July 2006.

U.S. Environmental Protection Agency (EPA). Data Quality Assessment: Statistical Methods for Practitioners. EPA QA/G-9S 59; Washington, DC. February 2006.

U.S. Environmental Protection Agency. Test Methods for Evaluating Solid Waste, Physical/Chemical Methods, $3^{\text {rd }}$ Edition, Revision 6. Washington, DC. February 2007.

U.S. Nuclear Regulatory Commission (NRC). Minimum Detectable Concentrations with Typical Radiation Survey Instruments for Various Contaminants and Field Conditions NUREG-1507 Final; Washington, DC; 1997. 
APPENDIX A

DATA QUALIFIERS FOR CHEMICAL RESULTS 


\section{APPENDIX A \\ DATA QUALIFIERS FOR CHEMICAL RESULTS}

\section{DATA QUALIFIERS ${ }^{*}$}

$\mathbf{U}=$ Compound was analyzed for but not detected. The associated numerical value is the estimated sample quantitation limit which is included and corrected for dilution and percent moisture.

$\mathbf{J}=$ Indicates an estimated value. This flag is used under the following circumstances 1) when estimating a concentration for tentatively identified compounds (TICs) where a 1:1 response is assumed; or 2) when the mass spectral data indicate the presence of a compound that meets the identification criteria but the result is less than the specified detection limit but greater than zero. For example, if the limit of detection is $10 \mu \mathrm{g} / \mathrm{L}$ and a concentration of $3 \mu \mathrm{g} / \mathrm{L}$ is calculated, it is reported as $3 \mathrm{~J}$.

* Adapted from Lionville Laboratory, Inc TCE Analytical Data Package. 